\title{
Translation Map in Quantum Principal Bundles
}

\author{
Tomasz Brzeziński \\ Physique Nucléaire Théorique et Physique Mathématique \\ Université Libre de Bruxelles \\ Campus de la Plaine CP 229 \\ B-1050 Brussels, Belgium
}

February 1995

\begin{abstract}
The notion of a translation map in a quantum principal bundle is introduced. A translation map is then used to prove that the cross sections of a quantum fibre bundle $E(B, V, A)$ associated to a quantum principal bundle $P(B, A)$ are in bijective correspondence with equivariant maps $V \rightarrow P$, and that a quantum principal bundle is trivial if it admits a cross section which is an algebra map. The vertical automorphisms and gauge transformations of a quantum principal bundle are discussed. In particular it is shown that vertical automorphisms are in bijective correspondence with $\operatorname{Ad}_{\mathrm{R}}$-covariant maps $A \rightarrow$ $P$.
\end{abstract}

\footnotetext{
${ }^{*}$ Current address: Department of Applied Mathematics and Theoretical Physics, University of Cambridge, Cambridge CB3 9EW, U.K. E-mail: T.Brzezinski@damtp.cam.ac.uk
} 


\section{Introduction}

Quantum fibre bundles generalise the concept of a fibre bundle [12] which plays a vital rôle both in mathematics and physics [1, 10]. The main idea of this generalisation is to replace the algebra of functions on a structure group by a Hopf algebra [21] or a quantum group [7], and the algebras of functions on the base manifold, fibre etc. by non-commutative algebras. Such a generalisation of a fibre bundle was proposed by S. Majid and the author in [3]. The general considerations of [3] were illustrated by reference to an example of a canonical connection in the quantised Hopf bundle, i.e. by the deformation of a configuration that is known in physics as the Dirac monopole. The generalisation of fibre bundles proposed in [3] was then developed, partly independently, by various authors [18, 11, 8, 4, 5]. Although our work in [3] was motivated by physics, i.e. we aimed at a quantum group generalisation of gauge theories, the constructed objects seem to have more fundamental meaning reaching far beyond this particular application. It is therefore important not only to study physical constructions that may result from the quantum group generalisation of gauge theories but also to analyse the geometric structure of quantum fibre bundles.

In this paper we show that quantum fibre bundles enjoy some properties similar to the properties of classical fibre bundles. In particular we prove that cross sections of a quantum fibre bundle $E$ associated to a quantum principal bundle $P$ are in one-to-one correspondence with equivariant maps defined on the fibre of $E$ with values in $P$. We then deduce that if a quantum principal bundle has a cross section which is an algebra map, then the bundle is trivial. We also show how to interpret gauge transformations of a non-trivial quantum principal bundle in terms of vertical automorphisms, and how to identify them with the maps defined on a quantum structure group with values in $P$ and covariant under the adjoint coaction. All these results are in perfect correspondence with the classical situation, the only difference being that while in the case of a classical principal bundle gauge transformations may be viewed, via the above identifications, as sections of the associated adjoint bundle, in the case of quantum bundles such an interpretation is impossible because a quantum adjoint bundle does not exist.

In the classical case the above results are usually discussed in the context of locally trivial bundles and they are proved first for a trivial bundle and then deduced globally by patching trivial bundles together. Our experience in working with quantum fibre bundles tells us however that, although the notion of a locally trivial quantum bundle may be rigorously defined [3, 18], the resulting construction is not so natural as the classical one and usually leads to some technical difficulties. Therefore we prefer not to assume the local 
triviality of quantum bundles as long as possible and we employ a technique of proving of the above mentioned results that does not make use of the local structure of a bundle. The main tool that allows us to avoid the use of locally trivial bundles in this paper is a non-commutative generalisation of a translation map.

Classically, a translation map is defined as follows. Assume that we have a manifold with a free action of a Lie group $G$. Every two points on an orbit are then related by a unique element of $G$. A translation map assigns such an element of $G$ to any two points on an orbit. The notion of a principal bundle is equivalent to the existence of a continuous translation map [12, Section 4.2]. We construct the non-commutative version of a translation map by dualisation and show that the notion of a quantum principal bundle is equivalent to the existence of this generalised translation map. We then use this map throughout the paper to prove the above mentioned results.

Our paper is organised as follows. In Section 2 we briefly summarise the basic facts about Hopf algebras and quantum bundles. In Section 3 we define a translation map in a quantum principal bundle and analyse some of its properties. Section 4 is devoted to analysis of cross sections of a quantum fibre bundle. In Section 5, we identify vertical automorphisms of a quantum principal bundle with the maps covariant under the adjoint coaction, and give various equivalent descriptions of gauge transformations of a trivial quantum principal bundle. The paper ends with a computation of the gauge group for examples of bundles with finite structure groups in Section 6 .

\section{Preliminaries}

\subsection{Notation}

Here we summarise the notation we use in the sequel. $A$ denotes a Hopf algebra over a field $k$ of real or complex numbers, with a coproduct $\Delta: A \rightarrow A \otimes A$, counit $\epsilon: A \rightarrow k$ and the antipode $\mathrm{S}: A \rightarrow A$ [21]. For a coproduct we use an explicit expression $\Delta(a)=a_{(1)} \otimes a_{(2)}$, where the summation is implied according to the Sweedler sigma convention [21], i.e. $a_{(1)} \otimes a_{(2)}=\sum_{i \in I} a_{(1)}^{i} \otimes a_{(2)}^{i}$ for an index set $I$. We also use the notation

$$
a_{(1)} \otimes a_{(2)} \otimes \cdots \otimes a_{(n)}=(\Delta \otimes \underbrace{\mathrm{id} \otimes \cdots \otimes \mathrm{id}}_{n-2}) \circ \cdots \circ(\Delta \otimes \mathrm{id}) \circ \Delta
$$

which describes a multiple action of $\Delta$ on $a \in A$.

If $A$ is a Hopf algebra then $A^{*}$ denotes a dual Hopf algebra. $A^{*}$ has an algebra structure induced from the coalgebra structure of $A$ and a coalgebra structure induced from the 
algebra structure of $A$. For example $\forall x, y \in A^{*}, \forall a, b \in A,\langle x y, a\rangle=\left\langle x, a_{(1)}\right\rangle\left\langle y, a_{(2)}\right\rangle$, $\langle\Delta x, a \otimes b\rangle=\langle x, a b\rangle$ etc. , where $\langle\rangle:, A^{*} \otimes A \rightarrow k$ denotes the natural pairing.

Recall that a vector space $V$ is called a right $A$-comodule if there exists a linear map $\rho_{R}: V \rightarrow V \otimes A$, called a right coaction, such that $\left(\rho_{R} \otimes \mathrm{id}\right) \circ \rho_{R}=(\mathrm{id} \otimes \Delta) \circ \rho_{R}$ and $(\mathrm{id} \otimes \epsilon) \circ \rho_{R}=\mathrm{id}$. Similarly, a vector space $V$ is called a left $A$-comodule if there exists a linear map $\rho_{L}: V \rightarrow A \otimes V$, called a left coaction, such that $(\Delta \otimes \mathrm{id}) \circ \rho_{L}=\left(\operatorname{id} \otimes \rho_{L}\right) \circ \rho_{L}$ and $(\epsilon \otimes \mathrm{id}) \circ \rho_{L}=\mathrm{id}$. We say that a unital algebra $P$ over $k$ is a right $A$-comodule algebra if $P$ is a right $A$-comodule with a coaction $\Delta_{R}: P \rightarrow P \otimes A$, and $\Delta_{R}$ is an algebra map. The algebra structure of $P \otimes A$ is that of a tensor product algebra. For a coaction $\Delta_{R}$ we use an explicit notation $\Delta_{R} u=u^{(\overline{1})} \otimes u^{(\overline{2})}$, where the summation is also implied. Notice that $u^{(\overline{1})} \in P$ and $u^{(\overline{2})} \in A$.

If $P$ is a right $A$-comodule algebra then $P^{A}$ denotes a fixed point subalgebra of $P$, i.e. $P^{A}=\left\{u \in P: \Delta_{R} u=u \otimes 1\right\} . \quad P^{A}$ is a subalgebra of $P$ with a natural inclusion $j: P^{A} \hookrightarrow P$. In what follows we do not write this inclusion explicitly but it should be understood that the elements of $P^{A}$ are viewed as elements of $P$ via $j$.

Let $A$ be a Hopf algebra, $B$ be a unital algebra over $k$, and let $f, g: A \rightarrow B$ be linear maps. A convolution product of $f$ and $g$ is a linear map $f * g: A \rightarrow B$ given by $(f * g)(a)=f\left(a_{(1)}\right) g\left(a_{(2)}\right)$, for any $a \in A$. With respect to the convolution product, the set of all linear maps $A \rightarrow B$ forms an associative algebra with the unit $1_{B} \epsilon$. We say that a linear map $f: A \rightarrow B$ is convolution invertible if there is a map $f^{-1}: A \rightarrow B$ such that $f * f^{-1}=f^{-1} * f=1_{B} \epsilon$. The set of all convolution invertible maps $A \rightarrow B$ forms a multiplicative group. Similarly if $V$ is a right $A$-comodule and $f: V \rightarrow B$, $g: A \rightarrow B$ are linear maps then we define a convolution product $f * g: V \rightarrow B$ to be $(f * g)(v)=f\left(v^{(\overline{1})}\right) g\left(v^{(\overline{2})}\right)$.

In this paper we work with a universal differential structure [13, 14].

\subsection{Quantum Fibre Bundles}

In this section we recall the basic elements of the theory of quantum principal and associated bundles [3].

Let $A$ be a Hopf algebra, $P$ a right $A$-comodule algebra with a coaction $\Delta_{R}: P \rightarrow P \otimes A$. We define a map $\chi: P \otimes P \rightarrow P \otimes A$,

$$
\chi=(\cdot \otimes \mathrm{id}) \circ\left(\mathrm{id} \otimes \Delta_{R}\right) .
$$

Explicitly, $\chi(u \otimes v)=u v^{(\overline{1})} \otimes v^{(\overline{2})}$, for any $u, v \in P$. We say that the coaction $\Delta_{R}$ is free if $\chi$ is a surjection. We define also a map ${ }^{\sim}: P^{2} \rightarrow P \otimes \operatorname{ker} \epsilon$, by ${ }^{\sim}=\left.\chi\right|_{P^{2}}$. Here and 
below, for any algebra $P, P^{2} \subset P \otimes P$ denotes the kernel of the multiplication $\cdot$ in $P$. Let $B=P^{A}$. We say that the coaction $\Delta_{R}$ of $A$ on $P$ is exact, if ker ${ }^{\sim}=P B^{2} P$.

Definition 2.1 ([3]) Let $A$ be a Hopf algebra, $\left(P, \Delta_{R}\right)$ be a right $A$-comodule algebra and let $B=P^{A}$. We say that $P(B, A)$ is a quantum principal bundle with universal differential structure, with a structure quantum group $A$ and a base $B$ if the coaction $\Delta_{R}$ is free and exact.

The basic examples of quantum principal bundles are the trivial bundle $P(B, A, \Phi)$ with trivialisation $\Phi: A \rightarrow P$ [3, Example 4.2], and the bundle $P(B, A, \pi)$ [3, Lemma 5.2]. In the latter case $P$ and $A$ are Hopf algebras and $\pi: P \rightarrow A$ is a Hopf algebra projection, used in the construction of a quantum homogeneous space $B=P^{A}$. A large number of examples of quantum principal bundles $P(B, A, \pi)$ has been found recently in [16]

For a trivial quantum principal bundle $P(B, A, \Phi)$ one defines a gauge transformation as a convolution invertible map $\gamma: A \rightarrow B$ such that $\gamma(1)=1$. The set of all gauge transformations of $P(B, A, \Phi)$ forms a group with respect to the convolution product. This group is denoted by $\mathcal{A}(B)$. A map $\Psi: A \rightarrow P$ is a trivialisation of $P(B, A, \Phi)$ if and only if there exists $\gamma \in \mathcal{A}(B)$ such that $\Psi=\gamma * \Phi$.

Definition 2.2 (《3]) Let $P(B, A)$ be a quantum principal bundle and let $V$ be a right $A^{\mathrm{op}}$-comodule algebra, where $A^{\mathrm{op}}$ denotes the algebra which is isomorphic to $A$ as a vector space but has an opposite product, with coaction $\rho_{R}: V \rightarrow V \otimes A$. The space $P \otimes V$ is naturally endowed with a right $A$-comodule structure $\Delta_{E}: P \otimes V \rightarrow P \otimes V \otimes A$ given by $\Delta_{E}(u \otimes v)=u^{(\overline{1})} \otimes v^{(\overline{1})} \otimes u^{(\overline{2})} v^{(\overline{2})}$ for any $u \in P$ and $v \in V$. We say that the fixed point subalgebra $E$ of $P \otimes A$ with respect to $\Delta_{E}$ is a quantum fibre bundle associated to $P(B, A)$ over $B$ with structure quantum group $A$ and standard fibre $V$. We denote it by $E=E(B, V, A)$.1

\section{Definition and Properties of a Translation Map}

In this section we give a definition and analyse transformation properties of a translation map in a quantum principal bundle.

Definition 3.1 Let $P(B, A)$ be a quantum principal bundle. A linear map $\tau: A \rightarrow$ $P \otimes{ }_{B} P$, given by $\tau(a)=\sum_{i \in I} u_{i} \otimes{ }_{B} v_{i}$, where $\sum_{i \in I} u_{i} \otimes v_{i} \in \chi^{-1}(1 \otimes a)$, is called a translation map. We will often use an explicit notation $\tau(a)=\tau^{(1)}(a) \otimes_{B} \tau^{(2)}(a)$.

\footnotetext{
${ }^{\dagger}$ A slightly different definition of $E(B, V, A)$ was proposed in [1]. The formalism developed in this paper can be equally well applied to quantum fibre bundles in the sense of [11].
} 
Since $\chi$ is a surjection, $\tau$ is defined on the whole of $A$. Moreover, if $a=0$ then, by exactness of the coaction, the corresponding $\sum_{i \in I} u_{i} \otimes v_{i} \in P B^{2} P$. Hence $\sum_{i \in I} u_{i} \otimes_{B} v_{i}=0$ and the map $\tau$ is well-defined as a linear map. In fact, a translation map of Definition 3.1 is well-defined if and only if $P$ is a total space of a quantum principal bundle.

Lemma 3.2 Let $P$ be a right $A$-comodule algebra with a free coaction $\Delta_{R}: P \rightarrow P \otimes A$. Let $B=P^{A}$. If there is a translation map $\tau: A \rightarrow P \otimes_{B} P$ in $P$ then the coaction $\Delta_{R}$ is exact and hence there is a quantum principal bundle $P(B, A)$.

Proof. We need to show that if $\sum_{i \in I} u_{i} \otimes v_{i} \in \operatorname{ker}^{\sim}$ then $\sum_{i \in I} u_{i} \otimes v_{i} \in P B^{2} P$. Take any $\sum_{i \in I} u_{i} \otimes v_{i} \in$ ker $^{\sim}$ then $\sum_{i \in I} u_{i} \otimes v_{i} \in \chi^{-1}(1 \otimes 0)$. Since there is a translation map in $P$ we deduce that $\sum_{i \in I} u_{i} \otimes_{B} v_{i}=0$, what implies that $\sum_{i \in I} u_{i} \otimes v_{i} \in P B^{2} P$.

Definition 3.1 of a translation map reproduces exactly the classical definition 112, Definition 2.1], but in a language of algebras of functions on manifolds rather than manifolds themselves. Classically, if $X$ is a manifold on which a Lie group $G$ acts freely then the translation map $\hat{\tau}: X \times{ }_{M} X \rightarrow G$, where $M=X / G$, is defined by $u \hat{\tau}(u, v)=v$. Dualising this construction we arrive immediately at Definition 3.1.

Example 3.3 In a trivial quantum principal bundle $P(B, A, \Phi)$ the translation map is given by

$$
\tau(a)=\Phi^{-1}\left(a_{(1)}\right) \otimes_{B} \Phi\left(a_{(2)}\right) .
$$

Proof. Using the fact that the trivialisation $\Phi$ is an intertwiner, i.e. $\Delta_{R} \Phi=(\Phi \otimes$ id $) \Delta$, and that $\Phi(1)=1$ we obtain

$$
\begin{aligned}
\chi\left(\Phi^{-1}\left(a_{(1)}\right) \otimes \Phi\left(a_{(2)}\right)\right) & =\Phi^{-1}\left(a_{(1)}\right) \Phi\left(a_{(2)}\right)^{(\overline{1})} \otimes \Phi\left(a_{(2)}\right)^{(\overline{2})} \\
& =\Phi^{-1}\left(a_{(1)}\right) \Phi\left(a_{(2)}\right) \otimes a_{(3)}=1 \otimes a,
\end{aligned}
$$

for any $a \in A$. Hence the map $\tau$ given by Eq. (2) is a translation map as stated.

Example 3.4 In a quantum principal bundle $P(B, A, \pi)$ on a quantum homogeneous space $B$ the translation map $\tau: A \rightarrow P \otimes_{B} P$ is given by

$$
\tau(a)=\mathrm{S} u_{(1)} \otimes_{B} u_{(2)}
$$

where $u \in \pi^{-1}(a)$. 
Proof. For any $a \in A$ we apply the map $\chi$ to $\mathrm{S} u_{(1)} \otimes u_{(2)}$, where $u \in \pi^{-1}(a)$, to obtain

$$
\chi\left(\mathrm{S} u_{(1)} \otimes u_{(2)}\right)=\left(\mathrm{S} u_{(1)}\right) u_{(2)}^{(\overline{1})} \otimes u_{(2)}^{(\overline{2})}=\left(\mathrm{S} u_{(1)}\right) u_{(2)} \otimes \pi\left(u_{(3)}\right)=1 \otimes \pi(u)=1 \otimes a .
$$

We conclude that $\tau$ given by Eq. (3) is a translation map as stated.

Before we analyse some properties of a translation map in a quantum principal bundle we study the behaviour of the map $\chi$, given by Eq. (1), with respect to the coaction $\Delta_{R}$. Firstly we observe that if $P$ is a right $A$-comodule then also $P \otimes P$ is a right $A$-comodule with a coaction $\Delta_{R}^{\otimes}=(\mathrm{id} \otimes \mathrm{id} \otimes \cdot) \circ\left(\mathrm{id} \otimes \sigma_{P} \otimes \mathrm{id}\right) \circ\left(\Delta_{R} \otimes \Delta_{R}\right)$, where $\sigma_{P}: P \otimes P \rightarrow P \otimes P$ is a twist map $\sigma_{P}: u \otimes v \mapsto v \otimes u$. Explicitly, $\Delta_{R}^{\otimes}(u \otimes v)=u^{(\overline{1})} \otimes v^{(\overline{1})} \otimes u^{(\overline{2})} v^{(\overline{2})}$. Secondly, both $P$ and $A$ are right $A$-comodules with the coactions $\Delta_{R}: P \rightarrow P \otimes A$ and $\operatorname{Ad}_{\mathrm{R}}: A \rightarrow A \otimes A$, $\operatorname{Ad}_{\mathrm{R}}(a)=a_{(2)} \otimes\left(\mathrm{S} a_{(1)}\right) a_{(3)}$. Therefore $P \otimes A$ is a right $A$-comodule with the coaction

$$
\Delta_{R}^{\mathrm{ad}}=(\mathrm{id} \otimes \mathrm{id} \otimes \cdot) \circ\left(\mathrm{id} \otimes \sigma_{P A} \otimes \mathrm{id}\right) \circ\left(\Delta_{R} \otimes \mathrm{Ad}_{\mathrm{R}}\right)
$$

where $\sigma_{P A}: P \otimes A \rightarrow A \otimes P$ is a twist map.

Finally we define a linear map $\nu: P \otimes A \rightarrow A \otimes P \otimes A, \nu: u \otimes a \mapsto u^{(\overline{2})} \mathrm{S}_{(1)} \otimes u^{(\overline{1})} \otimes a_{(2)}$. Now we can prove the following lemma.

Lemma 3.5 Let $P$ be a right $A$-comodule algebra and let $\chi: P \otimes P \rightarrow P \otimes A$ be given by (1). Then

1. $(\mathrm{id} \otimes \chi) \circ\left(\sigma_{P A} \circ \Delta_{R} \otimes \mathrm{id}\right)=\nu \circ \chi ;$

2. $(\chi \otimes \mathrm{id}) \circ\left(\mathrm{id} \otimes \Delta_{R}\right)=(\mathrm{id} \otimes \Delta) \circ \chi$;

3. $(\chi \otimes \mathrm{id}) \circ \Delta_{R}^{\otimes}=\Delta_{R}^{\mathrm{ad}} \circ \chi$.

Proof. Since all the maps discussed in this lemma are linear it suffices to prove the required equalities for any $u \otimes v \in P \otimes P$. To prove the first assertion we compute

$$
(\mathrm{id} \otimes \chi) \circ\left(\sigma_{P A} \circ \Delta_{R} \otimes \mathrm{id}\right)(u \otimes v)=(\mathrm{id} \otimes \chi)\left(u^{(\overline{2})} \otimes u^{(\overline{1})} \otimes v\right)=u^{(\overline{2})} \otimes u^{(\overline{1})} v^{(\overline{1})} \otimes v^{(\overline{2})} .
$$

On the other hand

$$
\nu \circ \chi(u \otimes v)=\nu\left(u v^{(\overline{1})} \otimes v^{(\overline{2})}\right)=u^{(\overline{2})} v^{(\overline{2})}{ }_{(1)} \mathrm{S} v^{(\overline{2})}{ }_{(2)} \otimes u^{(\overline{1})} v^{(\overline{1})} \otimes v^{(\overline{2})}{ }_{(3)}=u^{(\overline{2})} \otimes u^{(\overline{1})} v^{(\overline{1})} \otimes v^{(\overline{2})} .
$$

Thus

$$
(\mathrm{id} \otimes \chi) \circ\left(\sigma_{P A} \circ \Delta_{R} \otimes \mathrm{id}\right)=\nu \circ \chi
$$

and the first assertion of the lemma holds. 
The second assertion follows from the definition of $\chi$ and the fact that $\Delta_{R}$ is a coaction. Explicitly,

$$
\begin{aligned}
(\chi \otimes \mathrm{id}) \circ\left(\mathrm{id} \otimes \Delta_{R}\right) & =(\cdot \otimes \mathrm{id} \otimes \mathrm{id}) \circ\left(\mathrm{id} \otimes \Delta_{R} \otimes \mathrm{id}\right) \circ\left(\mathrm{id} \otimes \Delta_{R}\right) \\
& =(\cdot \otimes \mathrm{id} \otimes \mathrm{id}) \circ(\mathrm{id} \otimes \mathrm{id} \otimes \Delta) \circ\left(\mathrm{id} \otimes \Delta_{R}\right) \\
& =(\mathrm{id} \otimes \Delta) \circ(\cdot \otimes \mathrm{id}) \circ\left(\mathrm{id} \otimes \Delta_{R}\right)=(\mathrm{id} \otimes \Delta) \circ \chi
\end{aligned}
$$

Finally, the third assertion was proven in [3, Lemma 4.3]. It is also a consequence of the first two assertions.

Now we can state the proposition that collects the transformation properties of a translation map in a quantum principal bundle.

Proposition 3.6 The translation map $\tau: A \rightarrow P \otimes_{B} P$ in a quantum principal bundle $P(B, A)$ has the following properties:

1. $\left(\sigma_{P A} \circ \Delta_{R} \otimes_{B}\right.$ id $) \circ \tau=(\mathrm{S} \otimes \tau) \circ \Delta$;

2. $\left(\mathrm{id} \otimes_{B} \Delta_{R}\right) \circ \tau=(\tau \otimes \mathrm{id}) \circ \Delta$;

3. $\Delta_{R}^{\otimes} \circ \tau=(\tau \otimes \mathrm{id}) \circ \operatorname{Ad}_{\mathrm{R}}$

4. $\cdot \circ \tau=1 \epsilon$.

Proof. 1. Let $\tau(a)=\tau^{(1)}(a) \otimes_{B} \tau^{(2)}(a)$ for any $a \in A$. The first assertion of Lemma 3.5 yields

$$
(\mathrm{id} \otimes \chi) \circ\left(\sigma_{P A} \circ \Delta_{R} \otimes \mathrm{id}\right)\left(\tau^{(1)}(a) \otimes \tau^{(2)}(a)\right)=\mathrm{S} a_{(1)} \otimes 1 \otimes a_{(2)} .
$$

Since $\chi\left(\tau^{(1)}(a) \otimes \tau^{(2)}(a)\right)=1 \otimes a$ for any $a \in A$ we immediately deduce that

$$
\left(\sigma_{P A} \circ \Delta_{R} \otimes_{B} \text { id }\right) \circ \tau=(\mathrm{S} \otimes \tau) \circ \Delta .
$$

2. Using the second assertion of Lemma 3.5 we obtain

$$
\begin{aligned}
\left(\mathrm{id} \otimes_{B} \Delta_{R}\right) \circ \tau(a) & =\tau^{(1)}(a) \otimes_{B} \tau^{(2)}(a)^{(\overline{1})} \otimes \tau^{(2)}(a)^{(\overline{2})} \\
& =\tau^{(1)}\left(a_{(1)}\right) \otimes_{B} \tau^{(2)}\left(a_{(1)}\right) \otimes a_{(2)}=\tau\left(a_{(1)}\right) \otimes a_{(2)},
\end{aligned}
$$

i.e. the assertion.

3. The third assertion of Lemma 3.5 yields for any $a \in A$

$$
(\chi \otimes \mathrm{id}) \circ \Delta_{R}^{\otimes}\left(\tau^{(1)}(a) \otimes \tau^{(2)}(a)\right)=1 \otimes \operatorname{Ad}_{\mathrm{R}}(a)=1 \otimes a_{(2)} \otimes \mathrm{S} a_{(1)} a_{(3)} .
$$

Hence, using the definition of $\tau$, we immediately find that

$$
\Delta_{R}^{\otimes} \circ \tau=(\tau \otimes \mathrm{id}) \circ \operatorname{Ad}_{\mathrm{R}} .
$$


4. For any $a \in A$ we have

$$
\tau^{(1)}(a) \tau^{(2)}(a)^{(\overline{1})} \otimes \tau^{(2)}(a)^{(\overline{2})}=1 \otimes a .
$$

Applying id $\otimes \epsilon$ to both sides of Eq. (四) we immediately obtain the assertion. This ends the proof of the proposition.

\section{Cross Sections of a Quantum Fibre Bundle}

In this section we use the notion of a translation map in a quantum principal bundle $P(B, A)$ to identify cross sections of a quantum fibre bundle $E(B, V, A)$ with equivariant maps $V \rightarrow P$. Recall that a linear map $\phi: V \rightarrow P$ is said to be equivariant if $\Delta_{R} \phi=$ ( $\phi \otimes$ id) $\rho_{R}$, where $\rho_{R}$ is a right coaction of $A$ on $V$. In particular, our identification implies that a quantum principal bundle is trivial if it admits a cross section which is an algebra map. We begin with the following definition.

Definition 4.1 Let $E(B, V, A)$ be a quantum fibre bundle associated to a quantum principal bundle $P(B, A)$. A left $B$-module map $s: E \rightarrow B$ such that $s(1)=1$ is called a cross section of $E(B, V, A)$. The set of cross sections of $E(B, V, A)$ is denoted by $\Gamma(E)$.

Lemma 4.2 If $s: E \rightarrow B$ is a cross section of a quantum fibre bundle $E(B, V, A)$ then $s \circ j_{E}=\mathrm{id}$, where $j_{E}: B \hookrightarrow E$ is a natural inclusion $j_{E}: b \mapsto b \otimes 1_{V}$.

Proof. For any $b \in B, s \circ j_{E}(b)=s(b \otimes 1)=b s(1)=b$.

The result of trivial Lemma 4.2 justifies the term cross section used in Definition 4.1 . We remark that in [0] cross sections of a quantum fibre bundle were defined as maps $E \rightarrow B$ having the property described in Lemma 4.2. Definition 4.1 is more restrictive than that of [3] since the fact that $s \circ j_{E}=$ id does not imply that $s$ is a left $B$-module map. We also remark that the definition of a cross section of a quantum fibre bundle analogous to the one we use here was first proposed in [11].

Now we can state the first of two main results of this section.

Theorem 4.3 Let $A$ be a Hopf algebra with a bijective antipode. Cross sections of a quantum fibre bundle $E(B, V, A)$ associated to a quantum principal bundle $P(B, A)$ are in bijective correspondence with equivariant maps $\phi: V \rightarrow P$ such that $\phi(1)=1$. 
Proof. The fact that each equivariant map $\phi: V \rightarrow P$ induces a map $s$ such that $s \circ j_{E}=\mathrm{id}$, by $s=\cdot(\mathrm{id} \otimes \phi)$ was proved in [3], Proposition A.5]. Clearly, $s(b x)=b s(x)$ for any $b \in B$ and $x \in E$, and $\mathrm{s}(1)=1$, hence $s$ defined above is a cross section of $E(B, V, A)$.

Conversely, for any $s \in \Gamma(E)$ we define a map $\phi: V \rightarrow P$ by

$$
\phi: v \mapsto \tau^{(1)}\left(\mathrm{S}^{-1} v^{(\overline{2})}\right) s\left(\tau^{(2)}\left(\mathrm{S}^{-1} v^{(\overline{2})}\right) \otimes v^{(\overline{1})}\right),
$$

where $\tau(a)=\tau^{(1)}(a) \otimes_{B} \tau^{(2)}(a)$ is a translation map in $P(B, A)$. We observe that this definition of $\phi$ makes sense since $s$ is a $B$-module map and, by Proposition 3.6, $\forall v \in V$,

$$
\tau\left(\mathrm{S}^{-1} v^{(\overline{2})}\right) \otimes v^{(\overline{1})} \in P \otimes_{B} E .
$$

Explicitly,

$$
\begin{aligned}
\left(\mathrm{id} \otimes_{B} \Delta_{E}\right)\left(\tau\left(\mathrm{S}^{-1} v^{(\overline{2})}\right) \otimes v^{(\overline{1})}\right)= & \tau^{(1)}\left(\mathrm{S}^{-1} v^{(\overline{2})}{ }_{(2)}\right) \otimes_{B} \tau^{(2)}\left(\mathrm{S}^{-1} v^{(\overline{2})}{ }_{(2)}\right)^{(\overline{1})} \otimes v^{(\overline{1})} \otimes \\
& \otimes \tau^{(2)}\left(\mathrm{S}^{-1} v^{(\overline{2})}(2)\right)^{(\overline{2})} v^{(\overline{2})}{ }_{(1)} \\
= & \tau^{(1)}\left(\mathrm{S}^{-1} v^{(\overline{2})}{ }_{(3)}\right) \otimes{ }_{B} \tau^{(2)}\left(\mathrm{S}^{-1} v^{(\overline{2})}{ }_{(3)}\right) \otimes v^{(\overline{1})} \otimes \\
& \otimes\left(\mathrm{S}^{-1} v^{(\overline{2})}{ }_{(2)}\right) v^{(\overline{2})}{ }_{(1)} \\
= & \tau\left(\mathrm{S}^{-1} v^{(\overline{2})}\right) \otimes v^{(\overline{1})} \otimes 1 .
\end{aligned}
$$

Clearly, $\phi(1)=1$. Furthermore, using Proposition 3.6 we find that for any $v \in V$

$$
\begin{aligned}
\Delta_{R} \phi(v) & =\Delta_{R}\left(\tau^{(1)}\left(\mathrm{S}^{-1} v^{(\overline{2})}\right) s\left(\tau^{(2)}\left(\mathrm{S}^{-1} v^{(\overline{2})}\right) \otimes v^{(\overline{1})}\right)\right. \\
& =\left(\tau^{(1)}\left(\mathrm{S}^{-1} v^{(\overline{2})}\right)^{(\overline{1})} s\left(\tau^{(2)}\left(\mathrm{S}^{-1} v^{(\overline{2})}\right) \otimes v^{(\overline{1})}\right) \otimes \tau^{(1)}\left(\mathrm{S}^{-1} v^{(\overline{2})}\right)^{(\overline{2})}\right. \\
& =\left(\tau^{(1)}\left(\mathrm{S}^{-1} v^{(\overline{2})}(1)\right)^{(\overline{1})} s\left(\tau^{(2)}\left(\mathrm{S}^{-1} v^{(\overline{2})}{ }_{(1)}\right) \otimes v^{(\overline{1})}\right) \otimes \mathrm{S} \circ \mathrm{S}^{-1} v^{(\overline{2})}(2)\right. \\
& =\phi\left(v^{(\overline{1})}\right) \otimes v^{(\overline{2})}=(\phi \otimes \mathrm{id}) \circ \rho_{R},
\end{aligned}
$$

hence $\phi$ is an equivariant map as required.

Therefore we have constructed the maps $\theta: \phi \mapsto \cdot(\operatorname{id} \otimes \phi)$ and $\tilde{\theta}: s \mapsto \phi$, where $\phi$ is given by Eq. (5). We now show that they are inverses to each other. For any $s \in \Gamma(E)$ and $\sum_{i \in I} u_{i} \otimes v_{i} \in E$ we have

$$
(\theta \circ \tilde{\theta})(s)\left(\sum_{i \in I} u_{i} \otimes v_{i}\right)=\sum_{i \in I} u_{i} \tilde{\theta}(s)\left(v_{i}\right)=\sum_{i \in I} u_{i} \tau^{(1)}\left(\mathrm{S}^{-1} v_{i}^{(\overline{2})}\right) s\left(\tau^{(2)}\left(\mathrm{S}^{-1} v_{i}^{(\overline{2})}\right) \otimes v_{i}^{(\overline{1})}\right) .
$$

Further, using Proposition 3.6 we find

$$
\begin{aligned}
& \left(\sigma_{P A} \circ \Delta_{R} \otimes_{B} \mathrm{id} \otimes \mathrm{id}\right)\left(\sum_{i \in I} u_{i} \tau^{(1)}\left(\mathrm{S}^{-1} v_{i}^{(\overline{2})}\right) \otimes_{B} \tau^{(2)}\left(\mathrm{S}^{-1} v_{i}^{(\overline{2})}\right) \otimes v_{i}{ }^{(\overline{1})}\right) \\
& \quad=\sum_{i \in I} u_{i}{ }^{(\overline{2})} v_{i}^{(\overline{2})}{ }_{(2)} \otimes u_{i}^{(\overline{1})} \tau^{(1)}\left(\mathrm{S}^{-1} v_{i}^{(\overline{2})}(1)\right) \otimes_{B} \tau^{(2)}\left(\mathrm{S}^{-1} v_{i}^{\left({ }^{(}\right)}(1)\right) \otimes v_{i}^{(\overline{1})} \\
& \quad=\mathrm{id} \otimes\left(\cdot \otimes_{B} \otimes \mathrm{id} \otimes \mathrm{id}\right) \circ\left(\mathrm{id} \otimes \tau \circ \mathrm{S}^{-1} \otimes \mathrm{id}\right) \circ\left(\mathrm{id} \otimes \sigma_{V A} \circ \rho_{R}\right)\left(\sum_{i \in I} u_{i}^{(\overline{2})} v_{i}^{(\overline{2})} \otimes u_{i}^{(\overline{1})} \otimes v_{i}^{(\overline{1})}\right) .
\end{aligned}
$$


Since $\sum_{i \in I} u_{i} \otimes v_{i} \in E$ we obtain

$$
\begin{aligned}
\left(\sigma_{P A} \circ \Delta_{R}\right. & \left.\otimes_{B} \mathrm{id} \otimes \mathrm{id}\right)\left(\sum_{i \in I} u_{i} \tau^{(1)}\left(\mathrm{S}^{-1} v_{i}{ }^{(\overline{2})}\right) \otimes_{B} \tau^{(2)}\left(\mathrm{S}^{-1} v_{i}{ }^{(\overline{2})}\right) \otimes v_{i}{ }^{(\overline{1})}\right) \\
& =\mathrm{id} \otimes\left(\cdot \otimes_{B} \otimes \mathrm{id} \otimes \mathrm{id}\right) \circ\left(\mathrm{id} \otimes \tau \circ \mathrm{S}^{-1} \otimes \mathrm{id}\right) \circ\left(\mathrm{id} \otimes \sigma_{V A} \circ \rho_{R}\right)\left(\sum_{i \in I} 1 \otimes u_{i} \otimes v_{i}\right) \\
& =\sum_{i \in I} 1 \otimes u_{i} \tau^{(1)}\left(\mathrm{S}^{-1} v_{i}{ }^{(\overline{2})}\right) \otimes_{B} \tau^{(2)}\left(\mathrm{S}^{-1} v_{i}{ }^{(\overline{2})}\right) \otimes v_{i}{ }^{(\overline{1})} .
\end{aligned}
$$

Hence

$$
\sum_{i \in I} u_{i} \tau^{(1)}\left(\mathrm{S}^{-1} v_{i}^{(\overline{2})}\right) \otimes_{B} \tau^{(2)}\left(\mathrm{S}^{-1} v_{i}^{(\overline{2})}\right) \otimes v_{i}^{(\overline{1})} \in B \otimes_{B} E .
$$

Therefore using the fact that $s$ is a left $B$-module map and Proposition 3.6 again, we obtain

$$
(\theta \circ \tilde{\theta})(s)\left(\sum_{i \in I} u_{i} \otimes v_{i}\right)=s\left(u_{i} \tau^{(1)}\left(\mathrm{S}^{-1} v_{i}^{(\overline{2})}\right) \tau^{(2)}\left(\mathrm{S}^{-1} v_{i}^{(\overline{2})}\right) \otimes v_{i}^{(\overline{1})}\right)=s\left(\sum_{i \in I} u_{i} \otimes v_{i}\right) .
$$

Conversely, for any $v \in V$ and equivariant $\phi: V \rightarrow P$

$$
\begin{aligned}
(\tilde{\theta} \circ \theta)(\phi)(v) & =\tau^{(1)}\left(\mathrm{S}^{-1} v^{(\overline{2})}\right) \theta(\phi)\left(\tau^{(2)}\left(\mathrm{S}^{-1} v^{(\overline{2})}\right) \otimes v^{(\overline{1})}\right) \\
& =\tau^{(1)}\left(\mathrm{S}^{-1} v^{(\overline{2})}\right) \tau^{(2)}\left(\mathrm{S}^{-1} v^{(\overline{2})}\right) \phi\left(v^{(\overline{1})}\right)=\phi(v) .
\end{aligned}
$$

Remark 4.4 There is a certain class of quantum fibre bundles [ [ 4 , Definition 11] for which Theorem 4.3 holds even if the antipode $\mathrm{S}: A \rightarrow A$ is not bijective. We consider a left $A$-comodule algebra with a coaction $\rho_{L}: V \rightarrow A \otimes V$. We view $V$ as a right $A^{o p}$-comodule algebra with a right coaction $\rho_{R}=(\operatorname{id} \otimes \mathrm{S}) \circ \sigma_{A V} \circ \rho_{L}$, where $\sigma_{A V}: A \otimes V \rightarrow V \otimes A$ is a twist map, and consider a quantum fibre bundle $E(B, V, A)$ associated to $P(B, A)$. In the case of such a bundle, to each cross-section $s$ we associate a map $\phi: V \rightarrow P$ given by $\phi=(\cdot \otimes \mathrm{id}) \circ\left(\mathrm{id} \otimes_{B} s\right) \circ(\tau \otimes \mathrm{id}) \circ \rho_{L}$ and proceed as in the proof of Theorem 1.3 to show that $s \mapsto \phi$ establishes the required bijective correspondence. $\diamond$

Example 4.5 Let $E(B, V, A)$ be a quantum fibre bundle associated to a trivial quantum principal bundle $P(B, A, \Phi)$. In this case every element of $E$ has the from $\sum_{i \in I} b_{i} \Phi_{E}\left(v_{i}\right)$, where $b_{i} \in B$ and $v_{i} \in V$, and $\Phi_{E}: V \rightarrow E, \Phi_{E}: v \mapsto \Phi\left(\mathrm{S}^{-1} v^{(\overline{2})}\right) \otimes v^{(\overline{1})}$ [包, Appendix]. The isomorphisms $\theta$ and $\theta^{-1}$ of the proof of Theorem $4.3 \mathrm{read}$

$$
\theta(\phi)\left(\sum_{i \in I} b_{i} \Phi_{E}\left(v_{i}\right)\right)=\sum_{i \in I} b_{i} \Phi\left(\mathrm{S}^{-1} v_{i}^{(\overline{2})}\right) \phi\left(v_{i}^{(\overline{1})}\right), \quad \theta^{-1}(s)(v)=\Phi^{-1}\left(\mathrm{~S}^{-1} v^{(\overline{2})}\right) s\left(\Phi_{E}\left(v^{(\overline{1})}\right)\right)
$$


for any equivariant $\phi: V \rightarrow P$ and $s \in \Gamma(E)$. Notice that the map $\theta^{-1}(s)$ obtained in this way is different from the equivariant map $\phi$ discussed in [3, Proposition A6].

If $E(B, V, A)$ is of the type described in Remark 4.4 then $\mathrm{S}^{-1}$ disappears from definitions of $\theta$ and $\theta^{-1}$. Explicitly we have

$$
\theta(\phi)\left(\sum_{i \in I} b_{i} \Phi_{E}\left(v_{i}\right)\right)=\sum_{i \in I} b_{i}(\Phi * \phi)\left(v_{i}\right), \quad \theta^{-1}(s)=\Phi^{-1} *\left(s \circ \Phi_{E}\right),
$$

where $*$ denotes convolution product between the maps $f: A \rightarrow P, g: V \rightarrow P$, given by $f * g=\cdot \circ(f \otimes g) \circ \rho_{L} \cdot \diamond$

Before we describe an important corollary of Theorem 4.3, we state a lemma that allows one to view a quantum principal bundle as a quantum fibre bundle.

Lemma 4.6 A quantum principal bundle $P(B, A)$ is a fibre bundle associated to $P(B, A)$ with the fibre which is isomorphic to $A$ as an algebra and with the coaction $\rho_{R}=(\mathrm{id} \otimes \mathrm{S}) \circ \Delta^{\prime}$, where $\Delta^{\prime}$ denotes the opposite coproduct, $\Delta^{\prime}(a)=a_{(2)} \otimes a_{(1)}$.

Proof. Firstly we show that the total space $E$ of the bundle constructed in the lemma is equal to $\operatorname{Im} \Delta_{R}$. We take any $u^{(\overline{1})} \otimes u^{(\overline{2})} \in \operatorname{Im} \Delta_{R}$. Then

$$
\Delta_{E}\left(u^{(\overline{1})} \otimes u^{(\overline{2})}\right)=u^{(\overline{1})} \otimes u_{(\overline{2})}^{(3)} \otimes u_{(1)}^{(\overline{2})} \mathrm{S} u^{(\overline{2})}{ }_{(2)}=u^{(\overline{1})} \otimes u^{(\overline{2})} \otimes 1,
$$

hence $u^{(\overline{1})} \otimes u^{(\overline{2})} \in E$. Conversely, let $\sum_{i \in I} u_{i} \otimes a^{i} \in E$. Then

$$
\sum_{i \in I} u_{i}^{(\overline{1})} \otimes a_{(2)}^{i} \otimes u_{i}^{(\overline{2})} \mathrm{S} a_{(1)}^{i}=\sum_{i \in I} u_{i} \otimes a^{i} \otimes 1 .
$$

Applying $\operatorname{id} \otimes \cdot \circ \sigma_{A}$, where $\sigma_{A}: A \otimes A \rightarrow A \otimes A$ is a twist map, to both sides of the above equation we obtain

$$
\sum_{i \in I} u_{i} \otimes a^{i}=\sum_{i \in I} \epsilon\left(a^{i}\right) u_{i}^{(\overline{1})} \otimes u_{i}^{(\overline{2})}
$$

hence $\sum_{i \in I} u_{i} \otimes a^{i}$ manifestly lies in $\operatorname{Im} \Delta_{R}$.

Secondly, we observe that $\operatorname{Im} \Delta_{R} \cong P$ as algebras, the isomorphism being provided by the coaction $\Delta_{R}$. Clearly $\Delta_{R}: P \rightarrow \operatorname{Im} \Delta_{R}$ is a surjection. Moreover, if $\Delta_{R} u=0$ then $u=(\mathrm{id} \otimes \epsilon) \Delta_{R} u=0$, hence $\operatorname{ker} \Delta_{R}=\{0\}$. Since $\Delta_{R}$ is an algebra map, the isomorphism is established. Therefore we may identify $E$ with $P$ and $P(B, A)$ is a quantum fibre bundle as stated.

Corollary 4.7 Cross sections $s: P \rightarrow B$ of a quantum principal bundle $P(B, A)$ are in bijective correspondence with the maps $\phi: A \rightarrow P$ such that $\Delta_{R} \phi=(\phi \otimes S) \Delta^{\prime}$ and $\phi(1)=1$. 
Proof. We identify $P(B, A)$ with a quantum fibre bundle $E(B, A, A)$ of Lemma 4.6 and then apply Theorem 4.3. When the total space $E=\operatorname{Im} \Delta_{R}$ is viewed back as $P$ then the bijective maps $\theta, \tilde{\theta}$ come out as $\theta: \phi \mapsto \mathrm{id} * \phi$ and $\tilde{\theta}: s \mapsto \cdot\left(\operatorname{id} \otimes_{B} s\right) \tau$. By this identification we see that we need not assume that the antipode is bijective since $\mathrm{S}$ does not enter the definition of $\tilde{\theta}$. On the other hand it also follows from the fact that $P(B, A)$ is a fibre bundle of the type described in Remark 4.4. One can also check directly that $\theta, \tilde{\theta}$ provide the required correspondence.

We notice that if $A$ has a bijective antipode $\mathrm{S}$, the sections of a quantum principal bundle $P(B, A)$ are in one-to-one correspondence with the maps $\psi: A \rightarrow P$ such that $\psi(1)=1$ and $\Delta_{R} \circ \psi=(\operatorname{id} \otimes \psi) \circ \Delta$. We simply need to define $\psi=\phi \circ \mathrm{S}^{-1}$, where $\phi$ is given by Corollary 4.7 .

Proposition 4.8 Any trivial quantum principal bundle $P(B, A, \Phi)$ admits a section. Conversely, if a bundle $P(B, A)$ admits a section which is an algebra map then $P(B, A)$ is trivial with the total space $P$ isomorphic to $B \otimes A$ as an algebra.

Proof. A convolution inverse of a trivialisation $\Phi$ of a trivial quantum principal bundle $P(B, A, \Phi)$ satisfies the assumptions of Corollary 4.7, hence $s=\mathrm{id} * \Phi^{-1}$ is a section of $P(B, A, \Phi)$. Conversely, assume that an algebra map $s: P \rightarrow B$ is a section of $P(B, A)$. Clearly, $s$ is a $B$-bimodule map, hence we can define a linear map $\Phi: A \rightarrow P, \Phi=$ $\cdot\left(s \otimes{ }_{B} \mathrm{id}\right) \circ \tau$. Let $\phi$ be a map $\tilde{\theta}(s)$ constructed in Corollary 4.7. We will show that $\Phi$ and $\phi$ are convolution inverses to each other. It will then follow that $\Phi$ is a trivialisation of $P(B, A)$. We have

$$
\begin{aligned}
\Phi\left(a_{(1)}\right) \phi\left(a_{(2)}\right) & =s\left(\tau^{(1)}\left(a_{(1)}\right)\right) \underbrace{\tau^{(2)}\left(a_{(1)}\right) \tau^{\prime(1)}\left(a_{(2)}\right)} s\left(\tau^{\prime(2)}\left(a_{(2)}\right)\right) \\
& =s\left(\tau^{(1)}\left(a_{(1)}\right)\right) s\left(\tau^{(2)}\left(a_{(1)}\right) \tau^{\prime(1)}\left(a_{(2)}\right) \tau^{\prime(2)}\left(a_{(2)}\right)\right) \\
& =s\left(\tau^{(1)}(a)\right) s\left(\tau^{(2)}(a)\right)=s\left(\tau^{(1)}(a) \tau^{(2)}(a)\right)=\epsilon(a) .
\end{aligned}
$$

The symbol $\tau^{\prime(1)}(a) \otimes \tau^{\prime(2)}(a)$ here denotes the second copy of $\tau(a)$ and we have used Proposition 3.6 to deduce that the expression in the brace is in B. Similarly,

$$
\begin{aligned}
\phi\left(a_{(1)}\right) \Phi\left(a_{(2)}\right) & =\tau^{(1)}\left(a_{(1)}\right) s\left(\tau^{(2)}\left(a_{(1)}\right)\right) s\left(\tau^{\prime(1)}\left(a_{(2)}\right)\right) \tau^{\prime(2)}\left(a_{(2)}\right) \\
& =\tau^{(1)}\left(a_{(1)}\right) s(\underbrace{\tau^{(2)}\left(a_{(1)}\right) \tau^{\prime(1)}\left(a_{(2)}\right)}) \tau^{\prime(2)}\left(a_{(2)}\right) \\
& =\tau^{(1)}\left(a_{(1)}\right) \tau^{(2)}\left(a_{(1)}\right) \tau^{\prime(1)}\left(a_{(2)}\right) \tau^{\prime(2)}\left(a_{(2)}\right)=\epsilon(a) .
\end{aligned}
$$

As before we have used Proposition 3.6 to deduce that the expression in the brace is in $B$. 
To prove that $P \cong B \otimes A$ as algebras we consider a map $\Theta: P \rightarrow B \otimes A, \Theta=(s \otimes$ id $) \circ$ $\Delta_{R}$. Clearly, $\Theta$ is an algebra map since both $s$ and $\Delta_{R}$ are algebra maps. Moreover the map $\tilde{\Theta}: P \rightarrow B \otimes A, \tilde{\Theta}: b \otimes a \mapsto b s\left(\tau^{(1)}(a)\right) \tau^{(2)}(a)$ is an inverse of $\Theta$. Explicitly,

$$
\Theta \circ \tilde{\Theta}(b \otimes a)=s\left(b s\left(\tau^{(1)}\left(a_{(1)}\right)\right) \tau^{(2)}\left(a_{(1)}\right)\right) \otimes a_{(2)}=b s\left(\tau^{(1)}\left(a_{(1)}\right)\right) s\left(\tau^{(2)}\left(a_{(1)}\right)\right) \otimes a_{(2)}=b \otimes a,
$$

and

$$
\begin{aligned}
\tilde{\Theta} \circ \Theta(u)= & s\left(u^{(\overline{1})}\right) s\left(\tau^{(1)}\left(u^{(\overline{2})}\right)\right) \tau^{(2)}\left(u^{(\overline{2})}\right)=s\left(u^{(\overline{1})} \tau^{(1)}\left(u^{(\overline{2})}\right)\right) \tau^{(2)}\left(u^{(\overline{2})}\right) \\
& =u^{(\overline{1})} \tau^{(1)}\left(u^{(\overline{2})}\right) \tau^{(2)}\left(u^{(\overline{2})}\right)=u
\end{aligned}
$$

Therefore we have obtained the criterion of triviality of a quantum principal bundle $P(B, A)$ which naturally generalises the classical case. In the classical limit all algebras are assumed commutative and all maps are algebra maps, hence Proposition 4.8 states simply that a classical principal bundle is trivial if and only if it admits a cross section.

We also remark that the criterion of triviality of a quantum principal bundle similar to the one in Proposition 4.8 was proved in [4, Theorem 2] in the case of locally trivial bundles. The locally trivial bundles used there have total spaces locally isomorphic to the tensor product algebras $B \otimes A$. We see that using the notion of a translation map we need not assume that a quantum principal bundle is locally trivial to prove Proposition 4.8. This fact reflects precisely the classical case in which the local triviality of a principal bundle is not necessary for validity of a classical version of Proposition 4.8 [12, Section 4.8].

Remark 4.9 We would like to emphasise that the existence of a cross section of a quantum principal bundle does not necessarily imply that the bundle is trivial. As an example of a non-trivial quantum principal bundle admitting a cross section we consider the quantum Hopf fibration $S U_{q}(2)\left(S_{q}^{2}, k\left[Z, Z^{-1}\right], \pi\right)$ [3, Section 5.2]. The total space of this bundle is the quantum group $S U_{q}(2)$, as an algebra generated by the identity and a matrix $T=$ $\left(t_{i j}\right)=\left(\begin{array}{ll}\alpha & \beta \\ \gamma & \delta\end{array}\right)$ [9]. The base space $S_{q}^{2} \subset S U_{q}(2)$ is a quantum two-sphere [19], defined as a fixed point subalgebra, $S_{q}^{2}=S U_{q}(2)^{k\left[Z, Z^{-1}\right]}$.

It was shown in [3] that $S U_{q}(2)\left(S_{q}^{2}, k\left[Z, Z^{-1}\right], \pi\right)$ is a non-trivial quantum principal bundle on the quantum homogeneous space $S_{q}^{2}$. We consider a linear map $\phi: k\left[Z, Z^{-1}\right] \rightarrow$ $S U_{q}(2)$, given by

$$
\phi(1)=1, \quad \phi\left(Z^{n}\right)=\delta^{n}, \quad \phi\left(Z^{-n}\right)=\alpha^{n},
$$

for any positive integer $n$. The map $\phi$ satisfies the hypothesis of Corollary 4.7, hence it induces a cross section $s: S U_{q}(2) \rightarrow S_{q}^{2}, s: u \mapsto u_{(1)} \phi\left(\pi\left(u_{(2)}\right)\right)$. One can clearly check, however, that $s$ is not an algebra map. 
Finally we notice that in the semi-classical limit, $q \rightarrow 1$, the quantum principal bundle $S U_{q}(2)\left(S_{q}^{2}, k\left[Z, Z^{-1}\right], \pi\right)$ reduces to the classical Hopf fibration written in terms of algebras of functions on manifolds. Also in this case the map $\phi$ above can be defined and hence the classical Hopf bundle admits a cross section $s$ in the sense of Definition 4.1. But since $s$ is not an algebra map, the cross section obtained in this way is not continuous. $\diamond$

\section{Vertical Automorphisms of a Quantum Principal Bundle}

Definition 5.1 Let $P(B, A)$ be a quantum principal bundle. Any left $B$-module automorphism $\mathcal{F}: P \rightarrow P$ such that $\mathcal{F}(1)=1$ and $\Delta_{R} \mathcal{F}=(\mathcal{F} \otimes \mathrm{id}) \Delta_{R}$ is called a vertical automorphism of the bundle $P(B, A)$. The set of all vertical automorphisms of $P(B, A)$ is denoted by $A u t_{B}(P)$.

Elements of $A u t_{B}(P)$ preserve both the base space $B$ and the action of the structure quantum group $A$ of a quantum principal bundle $P(B, A)$. $A_{u} t_{B}(P)$ can be equipped with a multiplicative group structure $:\left(\mathcal{F}_{1}, \mathcal{F}_{2}\right) \mapsto \mathcal{F}_{2} \circ \mathcal{F}_{1}$. Vertical automorphisms are often called gauge transformations and $\operatorname{Aut}_{B}(P)$ is termed a gauge group.

Proposition 5.2 Vertical automorphisms of a quantum principal bundle $P(B, A)$ are in bijective correspondence with convolution invertible maps $f: A \rightarrow P$ such that $f(1)=1$ and $\Delta_{R} f=(f \otimes \mathrm{id}) \mathrm{Ad}_{\mathrm{R}}$.

Proof. Let $f$ be a map satisfying the hypothesis of the proposition. Define a map $\mathcal{F}: P \rightarrow P$ by $\mathcal{F}=\mathrm{id} * f$. We show now that the map $\mathcal{F}$ is a vertical automorphism. First we need to prove that $\mathcal{F}$ is a left $B$-module map. Take any $b \in B$ and $u \in P$. Then

$$
\mathcal{F}(b u)=b u^{(\overline{1})} f\left(u^{(\overline{2})}\right)=b \mathcal{F}(u)
$$

hence the map $\mathcal{F}$ is a left $B$-module map as stated. To show that $\mathcal{F}$ is right-invariant we take any $u \in P$ and compute

$$
\begin{aligned}
\Delta_{R} \mathcal{F}(u) & =\Delta_{R}\left(u^{(\overline{1})} f\left(u^{(\overline{2})}\right)\right)=u^{(\overline{1})} f\left(u_{(3)}^{(\overline{2})}\right) \otimes u_{(1)}^{(\overline{2})}\left(\mathrm{S} u_{(2)}^{(\overline{2})}\right) u^{(\overline{2})}{ }_{(4)} \\
& =u^{(\overline{1})} f\left(u^{(\overline{2})}{ }_{(1)}\right) \otimes u^{(\overline{2})}{ }_{(2)}=(\mathcal{F} \otimes \mathrm{id}) \Delta_{R} u
\end{aligned}
$$

Finally we have to show that $\mathcal{F}$ is invertible. Consider a map $\tilde{\mathcal{F}}=\mathrm{id} * f^{-1}$. First we observe that $\Delta_{R} f^{-1}=\left(f^{-1} \otimes \mathrm{id}\right) \mathrm{Ad}_{\mathrm{R}}$. This implies that $\tilde{\mathcal{F}}$ is right-invariant. It is also a left $B$-module homomorphism. Now we use the right invariance of $\tilde{\mathcal{F}}$ to compute

$$
\mathcal{F} \circ \tilde{\mathcal{F}}=\tilde{\mathcal{F}} * f=\mathrm{id} * f^{-1} * f=\mathrm{id} .
$$


Similarly, by the right invariance of $\mathcal{F}$ we obtain $\tilde{\mathcal{F}} \circ \mathcal{F}=$ id. Therefore the map $\tilde{\mathcal{F}}$ is an inverse of $\mathcal{F}$ and the first part of the proposition is proven.

Conversely, for any $\mathcal{F} \in \operatorname{Aut}_{B}(P)$ we define a map $f: A \rightarrow P$,

$$
f=\cdot \circ\left(\mathrm{id} \otimes_{B} \mathcal{F}\right) \circ \tau
$$

where $\tau$ is a translation map. Explicitly $f(a)=\tau^{(1)}(a) \mathcal{F}\left(\tau^{(2)}(a)\right)$. The map $f$ is welldefined since $\mathcal{F}$ is a left $B$-module map. We need to show that $f$ given by Eq. (6) satisfies the assertion of the proposition. Clearly, $f(1)=1$. Next we derive the covariance property of $f$. We compute

$$
\begin{aligned}
\Delta_{R} f & =\Delta_{R} \circ \cdot \circ\left(\mathrm{id} \otimes_{B} \mathcal{F}\right) \circ \tau=(\cdot \otimes \mathrm{id}) \circ \Delta_{R}^{\otimes} \circ\left(\mathrm{id} \otimes_{B} \mathcal{F}\right) \circ \tau \\
& =(\cdot \otimes \mathrm{id}) \circ\left(\mathrm{id} \otimes_{B} \mathcal{F} \otimes \mathrm{id}\right) \circ \Delta_{R}^{\otimes} \circ \tau=\left(\cdot \circ\left(\mathrm{id} \otimes_{B} \mathcal{F}\right) \circ \tau \otimes \mathrm{id}\right) \circ \mathrm{Ad}_{\mathrm{R}}=(f \otimes \mathrm{id}) \mathrm{Ad}_{\mathrm{R}}
\end{aligned}
$$

where in the third equality we used that $\mathcal{F}$ is an intertwiner, and in the fourth one we used the assertion 3 of Proposition 3.6.

Consider a map $\tilde{f}: A \rightarrow P$, given by Eq. (6) but with $\mathcal{F}$ replaced by its inverse $\mathcal{F}^{-1}$. We show that the map $\tilde{f}$ is a convolution inverse of $f$. We have

$$
\tilde{f}\left(a_{(1)}\right) f\left(a_{(2)}\right)=\tau^{(1)}\left(a_{(1)}\right) \underbrace{\mathcal{F}^{-1}\left(\tau^{(2)}\left(a_{(1)}\right)\right) \tau^{\prime(1)}\left(a_{(2)}\right)} \mathcal{F}\left(\tau^{\prime(2)}\left(a_{(2)}\right)\right) .
$$

Using the assertions 1 and 2 of Proposition 3.6 we can easily see that the expression in the brace is in $B$. Since $\mathcal{F}$ is a left $B$-module map we obtain

$$
\begin{aligned}
\tilde{f}\left(a_{(1)}\right) f\left(a_{(2)}\right) & =\tau^{(1)}\left(a_{(1)}\right) \mathcal{F}\left(\mathcal{F}^{-1}\left(\tau^{(2)}\left(a_{(1)}\right)\right) \tau^{\prime(1)}\left(a_{(2)}\right) \tau^{\prime(2)}\left(a_{(2)}\right)\right) \\
& =\tau^{(1)}(a) \tau^{(2)}(a)=\epsilon(a) .
\end{aligned}
$$

To derive the second and third equalities we used the assertion 4 of Proposition 3.6. Similarly one proves that $f * \tilde{f}=\epsilon$.

Therefore we have established the correspondence between the vertical automorphisms and normalised, convolution invertible, $\operatorname{Ad}_{\mathrm{R}}$-covariant maps $f: A \rightarrow P$. We need to prove that this correspondence is bijective. Denote by $\theta_{A}$ the map $f \mapsto \mathrm{id} * f$, and by $\tilde{\theta}_{A}$ the map $\mathcal{F} \mapsto f$, where $f$ is given by Eq. (6). We will show that $\tilde{\theta}_{A}$ is an inverse of $\theta_{A}$. We have

$$
\tilde{\theta}_{A} \circ \theta_{A}(f(a))=\tilde{\theta}_{A}(\mathrm{id} * f)(a)=\tau^{(1)}(a)(\mathrm{id} * f)\left(\tau^{(2)}(a)\right)
$$

where $\tau^{(1)}(a) \otimes \tau^{(2)}(a) \in \chi^{-1}(1 \otimes a)$. But since $\tau^{(1)}(a) \tau^{(2)}(a)^{(\overline{1})} \otimes \tau^{(2)}(a)^{(\overline{2})}=1 \otimes a$, we obtain

$$
\tau^{(1)}(a)(\mathrm{id} * f)\left(\tau^{(2)}(a)\right)=\tau^{(1)}(a) \tau^{(2)}(a)^{(\overline{1})} f\left(\tau^{(2)}(a)^{(\overline{2})}\right)=f(a),
$$


hence $\tilde{\theta}_{A} \circ \theta_{A}(f)=f$.

Conversely, take any $\mathcal{F} \in A u t_{B}(P)$. Then

$$
\theta_{A} \circ \tilde{\theta}_{A}(\mathcal{F}(u))=\underbrace{u^{(\overline{1})} \tau^{(1)}\left(u^{(\overline{2})}\right)} \mathcal{F}\left(\tau^{(2)}\left(u^{(\overline{2})}\right)\right),
$$

for any $u \in P$. Using Proposition 3.6 we see that the expression in the brace is in $B$, hence, because $\mathcal{F}$ is a left $B$-module map,

$$
\theta_{A} \circ \tilde{\theta}_{A}(\mathcal{F}(u))=\mathcal{F}\left(u^{(\overline{1})} \tau^{(1)}\left(u^{(\overline{2})}\right) \tau^{(2)}\left(u^{(\overline{2})}\right)\right)=\mathcal{F}\left(u^{(\overline{1})} \epsilon\left(u^{(\overline{2})}\right)\right)=\mathcal{F}(u) .
$$

Therefore the map $\theta_{A}$ has an inverse and the proposition is proven.

It is easily seen from the proof of Proposition 5.2 that maps $f: A \rightarrow P$ form a group with respect to the convolution product. This group is denoted by $\mathcal{A}(P)$. In the classical case Proposition 5.2 allows one to interpret vertical automorphisms as cross sections of an associated adjoint bundle. This is because the elements of $\mathcal{A}(P)$ are in bijective correspondence with such cross sections by Proposition 4.7. In the general, non-commutative, situation there is no associated adjoint bundle and hence such an interpretation of vertical automorphisms is not possible. Still, as in the case of ordinary principal bundles, Proposition 5.2 implies the following:

Corollary 5.3 Aut $_{B}(P) \cong \mathcal{A}(P)$ as multiplicative groups.

Proof. In the proof of Proposition 5.2 we have defined the bijective map $\theta_{A}: \mathcal{A}(P) \rightarrow$ $\operatorname{Aut}_{B}(P), \theta_{A}: f \mapsto \mathrm{id} * f$. We need to show that $\theta_{A}$ is a group homomorphism. We take any $f_{1}, f_{2} \in \mathcal{A}(P)$ and compute

$$
\theta_{A}\left(f_{1} * f_{2}\right)=\mathrm{id} * f_{1} * f_{2}=\operatorname{id}\left(\theta_{A}\left(f_{1}\right)\right) * f_{2}=\theta_{A}\left(f_{2}\right) \circ \theta_{A}\left(f_{1}\right)=\theta_{A}\left(f_{1}\right) \theta_{A}\left(f_{2}\right) .
$$

From the proof of Proposition 5.2 it is clear that $\theta_{A}\left(f^{-1}\right)=\theta_{A}(f)^{-1}$. Furthermore, $\theta_{A}(1 \epsilon)=\mathrm{id} * \epsilon=\mathrm{id}$, hence the map $\theta_{A}$ is a group homomorphism.

Now we examine some properties of vertical automorphisms in the case of a trivial quantum principal bundle. In particular we identify vertical automorphisms of $P(B, A, \Phi)$ with gauge transformations.

Theorem 5.4 Let $P(B, A, \Phi)$ be a trivial quantum principal bundle. Then the groups Aut $_{B}(P), \mathcal{A}(P)$, and the gauge group $\mathcal{A}(B)$ are isomorphic to each other. If $A$ and $B$ are finite dimensional then the above groups are isomorphic to the group $\mathcal{R}(B)$ of all invertible elements $v \in B \otimes A^{*}$ such that $(\mathrm{id} \otimes \epsilon) v=1$. 
Proof. We prove the theorem in three steps.

1. Aut $(P) \cong \mathcal{A}(P)$. This isomorphism has already been proven in Corollary 5.3. We remark only that the map $\tilde{\theta}_{A}: A u t_{B}(P) \rightarrow \mathcal{A}(P)$, defined in the proof of Proposition 5.2 has the following simple form $\tilde{\theta}_{A}: \mathcal{F} \mapsto \Phi^{-1} *(\mathcal{F} \circ \Phi)$.

2. $\mathcal{A}(P) \cong \mathcal{A}(B)$. For any $\gamma \in \mathcal{A}(B)$ we consider a map $\theta_{B}(\gamma): A \rightarrow P$ given by

$$
\theta_{B}(\gamma)=\Phi^{-1} * \gamma * \Phi
$$

It is clear that the map $\theta_{B}(\gamma)$ is convolution invertible and is such that $\theta_{B}(\gamma)(1)=1$, and also

$$
\Delta_{R} \theta_{B}(\gamma)=\left(\theta_{B}(\gamma) \otimes \mathrm{id}\right) \operatorname{Ad}_{\mathrm{R}}
$$

hence $\theta_{B}(\gamma) \in \mathcal{A}(P)$. Now we show that the map $\theta_{B}: \mathcal{A}(B) \rightarrow \mathcal{A}(P)$ is a group homomorphism. Take any $\gamma_{1}, \gamma_{2} \in \mathcal{A}(B)$, then

$$
\theta_{B}\left(\gamma_{1} * \gamma_{2}\right)=\Phi^{-1} * \gamma_{1} * \gamma_{2} * \Phi=\Phi^{-1} * \gamma_{1} * \Phi * \Phi^{-1} * \gamma_{2} * \Phi=\theta_{B}\left(\gamma_{1}\right) * \theta_{B}\left(\gamma_{2}\right) .
$$

To complete the proof of the isomorphism it remains to construct an inverse of $\theta_{B}$. Consider a map $\tilde{\theta}_{B}: \mathcal{A}(P) \rightarrow \operatorname{Lin}(A, P)$ given by

$$
\tilde{\theta}_{B}(f)=\Phi * f * \Phi^{-1}
$$

for any $f \in \mathcal{A}(P)$. It is clear that for each $f \in \mathcal{A}(P), \tilde{\theta}_{B}(f)$ is a convolution invertible map such that $\tilde{\theta}_{B}(f)(1)=1$. Moreover,

$$
\begin{aligned}
\Delta_{R} \tilde{\theta}_{B}(f)(a) & =\Phi\left(a_{(1)}\right) f\left(a_{(3)}\right)^{(\overline{1})} \Phi^{-1}\left(a_{(5)}\right) \otimes a_{(2)} f\left(a_{(3)}\right)^{(\overline{2})} \mathrm{S} a_{(4)} \\
& =\Phi\left(a_{(1)}\right) f\left(a_{(4)}\right) \Phi^{-1}\left(a_{(7)}\right) \otimes a_{(2)}\left(\mathrm{S} a_{(3)}\right) a_{(5)} \mathrm{S} a_{(6)}=\tilde{\theta}_{B}(f)(a) \otimes 1 .
\end{aligned}
$$

This proves that $\tilde{\theta}_{B}(f) \in \mathcal{A}(B)$. It is now immediate that $\tilde{\theta}_{B}$ is an inverse of $\theta_{B}$. Explicitly,

$$
\left(\tilde{\theta}_{B} \circ \theta_{B}\right)(\gamma)=\tilde{\theta}_{B}\left(\Phi^{-1} * \gamma * \Phi\right)=\gamma
$$

and

$$
\left(\theta_{B} \circ \tilde{\theta}_{B}\right)(f)=\theta_{B}\left(\Phi * f * \Phi^{-1}\right)=f .
$$

Therefore the required isomorphism holds.

3. Finally we prove that if both $A$ and $B$ are finite dimensional algebras, then $\mathcal{A}(B) \cong$ $\mathcal{R}(B)$. We consider a map $\theta_{R}: \mathcal{R}(B) \rightarrow \operatorname{Lin}(A, B)$,

$$
\theta_{R}: v=\sum_{i \in I} v^{i} \otimes v_{i} \mapsto \gamma_{v}=\sum_{i \in I}<v_{i}, \cdot>v^{i},
$$


where $<,>: A^{*} \otimes A \rightarrow k$ denotes the natural pairing. It is clear that for each $v \in \mathcal{R}(B)$, $\gamma_{v}(1)=1$, since

$$
\gamma_{v}(1)=\sum_{i \in I}<v_{i}, 1>v^{i}=(\epsilon \otimes \mathrm{id}) v=1
$$

Also, $\gamma_{v}$ is a convolution invertible map with the convolution inverse $\gamma_{v}^{-1}=\gamma_{v^{-1}}$. Explicitly we have

$$
\begin{aligned}
\gamma_{v} * \gamma_{v^{-1}}(a) & =\sum_{i, j \in I}<v_{i}, a_{(1)}><\tilde{v}_{j}, a_{(2)}>v^{i} \tilde{v}^{j}=\sum_{i, j \in I}<v_{i} \tilde{v}_{j}, a>v^{i} \tilde{v}^{j} \\
& =<1, a>=\epsilon(a)
\end{aligned}
$$

where $v^{-1}=\sum_{j \in I} \tilde{v}^{j} \otimes \tilde{v}_{j}$. Similarly one shows that $\gamma_{v^{-1}} * \gamma_{v}=\epsilon$. Therefore for each $v \in$ $\mathcal{R}(B)$, we have that $\gamma_{v} \in \mathcal{A}(B)$. Now we need to prove that $\theta_{R}$ is a group homomorphism. For any $v, w \in \mathcal{R}(B)$ we compute

$$
\gamma_{v}\left(a_{(1)}\right) \gamma_{w}\left(a_{(2)}\right)=\sum_{i, j \in I}\left\langle v_{i}, a_{(1)}\right\rangle v^{i}\left\langle w_{j}, a_{(2)}\right\rangle w^{j}=\sum_{i, j \in I}\left\langle v_{i} w_{j}, a\right\rangle v^{i} w^{j}=\gamma_{v w}(a)
$$

For an inverse of $\theta_{R}$ we take a map $\tilde{\theta}_{R}: \mathcal{A}(B) \rightarrow \mathcal{R}(B)$ defined as follows. Let $\left\{b^{\beta} ; \beta \in \mathcal{B}\right\}$ be a basis of $B$. Then for any $\gamma \in \mathcal{A}(B)$ and any $a \in A$ we can write

$$
\gamma(a)=\sum_{\beta \in \mathcal{B}} \gamma_{\beta}(a) b^{\beta}
$$

where $\gamma_{\beta}(a) \in k$ are uniquely determined. Each $\gamma_{\beta}$ may be regarded as an element of $A^{*}$ such that $\gamma_{\beta}(a)=\left\langle\gamma_{\beta}, a\right\rangle$, for any $a \in A$. Since $\gamma_{\beta}$ are uniquely determined we can define a map

$$
\tilde{\theta}_{R}: \gamma \mapsto \sum_{\beta \in \mathcal{B}} b^{\beta} \otimes \gamma_{\beta}
$$

These are the elementary facts that if $\gamma \in \mathcal{A}(B)$, then $\tilde{\theta}_{R}(\gamma) \in \mathcal{R}(B)$ and that $\tilde{\theta}_{R}$ is an inverse of $\theta_{R}$.

This completes the proof of the theorem.

Therefore Theorem 5.4 allows one to interpret a vertical automorphism of a (locally) trivial quantum principal bundle as a change of local variables and truly as a gauge transformation of a trivial quantum principal bundle. Furthermore it gives a geometric interpretation of a universal $R$-matrix of a quasitriangular Hopf algebra $H$ [7] as a gauge transformation of the quantum frame bundle $D(H)^{*}(H, A)$ [3], Example 5.6], with a total space dual to Drinfeld's double $D(H)$ and the structure quantum group $A=H^{*}$ (cf. [2, Section 5.7.2]). 
Gauge transformations have also a natural interpretation in terms of isomorphisms of crossed product algebras. Recall from [3, 2] that a total space $P$ of a trivial quantum principal bundle $P(B, A, \Phi)$ is isomorphic to $B \otimes A$ as a vector space. Define the crossed product algebra $B_{\Phi} \rtimes A$ by equiping $B \otimes A$ with a multiplication

$$
\left(b_{1} \otimes a^{1}\right)\left(b_{2} \otimes a^{1}\right)=b_{1} \Phi\left(a_{(1)}^{1}\right) b_{2} \Phi\left(a_{(1)}^{2}\right) \Phi^{-1}\left(a_{(2)}^{1} a_{(2)}^{2}\right) \otimes a_{(3)}^{1} a_{(3)}^{2} .
$$

Then $P \cong B_{\Phi} \rtimes A$ as algebras. The isomorphism is given by $\theta_{\Phi}: u \mapsto u^{(\overline{1})} \Phi^{-1}\left(u^{(\overline{2})}(1)\right) \otimes$

$u^{(\overline{2})}(2)$. The following proposition is a special case of the result of Doi [6] (see also [15, Proposition 4.2]).

Proposition 5.5 Let $P(B, A, \Phi)$ be a trivial quantum principal bundle. Let for any trvialisation $\Psi$ of $P(B, A, \Phi), \Theta_{\Psi}: B_{\Psi} \rtimes A \rightarrow B_{\Phi} \rtimes A$ be a crossed product algebra isomorphism such that $\left.\Theta_{\Psi}\right|_{B}=\mathrm{id}$ and $\Delta_{R} \Theta_{\Psi}=\left(\Theta_{\Psi} \otimes \mathrm{id}\right) \Delta_{R}$. Then there is a bijective correspondence between all isomorphisms $\Theta_{\Psi}$ corresponding to all trivialisations $\Psi$ and the gauge transformations of $P(B, A, \Phi)$.

\section{An Example}

In this section we consider the simplest example of a trivial quantum principal bundle for which the gauge group can be computed explicitly. This example serves as an illustration of the considerations of the previous section and in particular of the use of Theorem 5.4 in computations of the gauge group. It also shows that the quantum gauge group $\mathcal{A}(B)$ of a classical trivial principal bundle is much bigger than the classical gauge group of this bundle. We assume that $k=\mathbf{C}$.

Proposition 6.1 Let $B$ be an $M$-dimensional semisimple algebra with unit and let $A=$ $\mathbf{C}[G]$ be a group algebra of a finite group $G$ of $N$ elements. Let $\rho_{l}, l=0, \ldots, L-1$ be all non-equivalent irreducible representations of $B$. Then for any trivial quantum principal bundle $P(B, A, \Phi)$,

$$
\mathcal{A}(B) \cong \underbrace{\mathcal{G} \oplus \mathcal{G} \oplus \ldots \oplus \mathcal{G}}_{N-1}
$$

where

$$
\mathcal{G}=\{b \in B ; b \text { is invertible in } B\}=\left\{b \in B ; \prod_{l=0}^{L-1} \operatorname{det} \rho_{l}(b) \neq 0\right\}
$$


Proof. Since the gauge group depends only on $A$ and $B$ it suffices to consider the case $P=B \otimes A$. Let $b_{0}=1, b_{1} \ldots, b_{M-1}$ be a basis of $B$, and $g_{0}=1, g_{1}, \ldots, g_{N-1}$ be elements of $G$. Assume that the multiplication in $B$ is given by

$$
b_{i} b_{j}=\sum_{k=0} N_{i j}^{k} b_{k}, \quad N_{i j}^{k} \in \mathbf{C} .
$$

First we show that the gauge group $\mathcal{A}(B)$ is a direct sum of identical groups. Let $\gamma \in \mathcal{A}(B)$ be given by $\gamma\left(g_{k}\right)=\sum_{i=0}^{M-1} a_{k}^{i} b_{i}$, for some $a_{k}^{i} \in \mathbf{C}$. By Theorem 5.4 there is a unique vertical automorphism $\mathcal{F}: B \otimes A \rightarrow B \otimes A$, canonically associated to $\gamma$. Since all generators of $A$ are group-like $\mathcal{F}$ can be easily computed,

$$
\mathcal{F}\left(b_{i} \otimes g_{k}\right)=\sum_{j, l=0}^{M-1} a_{k}^{j} N_{i j}^{l} b_{l} \otimes g_{k} .
$$

Since $\mathcal{F}$ does not act on elements of $G$, for each $k=0, \ldots, N-1$, it may be regarded as a linear automorphism of $B$. Noting that for $k=0, \mathcal{F}$ is the identity map we thus obtain

$$
\mathcal{A}(B) \cong \operatorname{Aut}_{B}(B \otimes A)=\underbrace{\mathcal{G} \oplus \mathcal{G} \oplus \ldots \oplus \mathcal{G}}_{N-1},
$$

where $\mathcal{G}$ may be identified with a group of all non-singular $M \times M$ matrices $F(\mathbf{a})=$ $\left(F(\mathbf{a})_{i}^{j}\right)_{i, j=0}^{M-1}$ with the entries

$$
F(\mathbf{a})_{i}^{j}=\sum_{k=0}^{M-1} a^{k} N_{i k}^{j} .
$$

Next we observe that the map $F: B \rightarrow \operatorname{End}(B), F: \sum_{i=0}^{M-1} a^{i} b_{i} \mapsto F(\mathbf{a})$ is a right regular representation of $B$. Since $B$ has a unit $F$ is an algebra isomorphism. Therefore the matrix $F(\mathbf{a})$ is non-singular if and only if $\sum_{i=0}^{M-1} a^{i} b_{i}$ is invertible. This gives the first description of $\mathcal{G}$. Secondly we observe that because $B$ is semisimple $F$ is completely reducible and it contains every irreducible representation of $B$ at least once. Therefore $\operatorname{det} F(\mathbf{a}) \neq 0$ iff $\prod_{l=0}^{L-1} \operatorname{det} \rho_{l}\left(\sum_{i=0}^{M-1} a^{i} b_{i}\right) \neq 0$. This establishes the second description of $\mathcal{G}$.

Example 6.2 Let $P=\mathbf{C}\left[\mathbf{Z}_{M N}\right] \equiv \mathbf{C}[1, g] /\left(g^{M N}-1\right)$ and $A=\mathbf{C}\left[\mathbf{Z}_{N}\right] \equiv \mathbf{C}[1, h] /\left(h^{N}-1\right)$ have the standard Hopf algebra structure, i.e. $\Delta g=g \otimes g, \Delta h=h \otimes h$ etc. Define a Hopf algebra projection $\pi: P \rightarrow A, \pi: g \mapsto h$. Let $\Delta_{R}: P \rightarrow P \otimes A$ be a right coaction given by a pushout $\Delta_{R}=(\mathrm{id} \otimes \pi) \circ \Delta$. Then $B=P^{A}$ is a subalgebra of $P$ as a vector space spanned by $\left\{1, g^{N}, \ldots, g^{N(M-1)}\right\}$ and hence is isomorphic to $\mathbf{C}\left[\mathbf{Z}_{M}\right] . \quad B$ is a semisimple algebra with unit as a group algebra. Clearly $P(B, A, \pi)$ is a trivial quantum principal bundle on the homgeneous space $B$ with trivialisation given by $\Phi_{0}: A \rightarrow P, \Phi_{0}: h^{n} \mapsto g^{n}$, $0 \leq n \leq N-1$. 
Since $B$ is a commutative algebra all the irreducible representations $\rho_{k}, k=0, \ldots, M-1$ of $B$ are one dimensional,

$$
\rho_{k}\left(g^{m N}\right)=e^{\frac{2 \pi k m}{M} i}
$$

Therefore $b=a_{m} g^{m N}$ is an element of $\mathcal{G}$ iff $\forall k \in\{0, \ldots, M-1\}, \sum_{m=0}^{M-1} a_{m} e^{\frac{2 \pi k m}{M} i} \neq 0$.

To gain the further inside into the structure of $\mathcal{G}$ we consider the group homomorphism $\rho: \mathcal{G} \rightarrow\left(\mathbf{C}^{*}\right)^{M}=\mathbf{C}^{*} \oplus \ldots \oplus \mathbf{C}^{*}, \rho: b \mapsto\left(\rho_{0}(b), \ldots, \rho_{M-1}(b)\right)$. Let $b=\sum_{m=0}^{M-1} a_{m} g^{m N}$ and $c_{k}=\sum_{m=0}^{M-1} a_{m} e^{\frac{2 \pi k m}{M} i}=\rho_{k}(b)$. Clearly the map $\rho$ is injective. It is also surjective since the Vandermonde determinant $\operatorname{det}\left(e^{\frac{2 \pi k m}{M}}\right)_{k, m=0}^{M-1}$ is non-zero. Therefore $\rho$ is an isomorphism of multiplicative groups, $\mathcal{G} \cong\left(\mathbf{C}^{*}\right)^{M}$, and $\mathcal{A}(B)=\left(\mathbf{C}^{*}\right)^{M(N-1)}$.

Using the properties of the Vandermonde determinants [17, pp. 322 and 333] one easily finds that the inverse of $\rho, \rho^{-1}:\left(c_{0}, \ldots, c_{M-1}\right) \mapsto \sum_{m=0}^{M-1} a_{m} g^{m N}$ is given by

$$
a_{m}=(-1)^{m} \sum_{k=0}^{M-1} \prod_{\substack{j=0 \\ j \neq k}}^{M-1}\left(e^{2 \pi \frac{j}{M} i}-e^{2 \pi \frac{k}{M} i}\right)^{-1} \sum_{\mathbf{m}} \delta_{0 m_{k}} c_{k} \exp \left(2 \pi i \frac{1}{M} \sum_{l=1}^{M-1} l m_{l}\right)
$$

where the second sum runs over all sequences $\mathbf{m}=\left(m_{0}, \ldots, m_{M-1}\right), m_{l}=0,1$, such that $m_{0}+\ldots+m_{M-1}=M-1-m$.

The quantum principal bundle discussed in this example may serve as an illustration of the fact that the quantum gauge group $\mathcal{A}(B)$ of a classical principal bundle is much bigger that the classical gauge group $\mathcal{A}_{\text {alg }}(B)$, whose elements are algebra maps $A \rightarrow B$.

To compute $\mathcal{A}_{\text {alg }}(B)$ we notice first that a gauge transformation $\gamma \in \mathcal{A}_{\text {alg }}(B)$ is fully determined by its action on $h$, hence $\mathcal{A}(B)_{\text {alg }}$ may be identified with a subgroup of $\mathcal{G}=\left(\mathbf{C}^{*}\right)^{M}$. Secondly $\gamma(h)^{N}=1$ because $\gamma$ is an algebra map. When $\gamma$ is viewed as $\left(c_{0}, \ldots, c_{M-1}\right) \in\left(\mathbf{C}^{*}\right)^{M}$ via the isomorphism $\rho$, the condition $\gamma(h)^{N}=1$ is equivalent to $c_{k}^{N}=1$. Hence each $c_{k}$ is an element of $\mathbf{Z}_{N}$ viewed as a multiplicative subgroup of $\mathbf{C}^{*}$ by $\mathbf{Z}_{N} \ni n \mapsto e^{2 \pi n i / N} \in \mathbf{C}^{*}$. Therefore

$$
\mathcal{A}_{\text {alg }}(B) \cong \underbrace{\mathbf{Z}_{N} \oplus \mathbf{Z}_{N} \oplus \ldots \oplus \mathbf{Z}_{N}}_{M}
$$

Finally we can show that the bundle $P(B, A, \pi)$ of Example 6.2 is a classical trivial bundle in the sense that it admits a trivialisation which is an algebra map. Classifying all such trivialisations we classify all cross-sections of $P(B, A, \pi)$ which are algebra maps by Proposition 4.8.

The algebraic trivialisation $\Phi$ is fully determined by its action on $h$ and also it must be related to $\Phi_{0}$ by a gauge transformation. Therefore we must have $\Phi(h)=\sum_{m=0}^{M-1} a_{m} g^{m N+1}$, for some $a_{m} \in \mathbf{C}$. The trivialisation $\Phi$ is an algebra map if and only if $\Phi(h)^{N}=1$. 
This condition may be easily solved if we first notice that it must be satisfied in all representations $\rho_{k}$ and then linearise obtained system of equations by taking its $N$-th root. As a result we obtain the $N^{M}$ systems of $M$ linear equations parametrised by the sequences $\left(n_{0}, \ldots, n_{M-1}\right), n_{k}=0, \ldots, N-1$. Each such system may be easily solved using the properties of the Vandermonde determinants. We obtain

$$
a_{m}=(-1)^{m} \sum_{k=0}^{M-1} \prod_{\substack{j=0 \\ j \neq k}}^{M-1}\left(e^{2 \pi i \frac{j}{M}}-e^{2 \pi i \frac{k}{M}}\right)^{-1} \sum_{\mathbf{m}} \delta_{0 m_{k}} \exp \left(2 \pi i\left(\frac{1}{M} \sum_{l=1}^{M-1} l m_{l}+\frac{n_{k}}{N}-\frac{k}{M N}\right)\right)
$$

where the range of the second sum is over all sequences $\mathbf{m}=\left(m_{0}, \ldots, m_{M-1}\right)$ as in (10).

We notice that for some choices of $M$ and $N$, for example $M=N=2$, there are no real solutions $a_{m}$ to the equation $\Phi(h)^{N}=1$. Therefore in these cases the bundle $P(B, A, \pi)$ can be considered as a classical trivial bundle over $\mathbf{C}$ and as a trivial quantum bundle and nontrivial classical bundle over $\mathbf{R}$. $\diamond$

Remark 6.3 Interesting examples of commutative semisimple algebras $B$ come from the fusion rings of algebraic quantum field theories. A fusion ring is a ring with involution generated by $b_{i}$ subject to the relation (8) with $N_{i j}^{k} \in \mathbf{Z}$. To each $b_{i}$ one can associate a nonnegative number $d\left(b_{i}\right)$ which has a meaning of a quantum dimension of a representation of an internal braided (or quasi-quantum) symmetry group, corresponding to $b_{i}$. By the theorem of Rehren [20, Proposition 3.3] an element $b_{i}$ of a fusion ring is invertible iff $d\left(b_{i}\right)=1$. We can extend fusion ring to an algebra $B$ over $\mathbf{C}$ and we can extend linearly the function $d$ to $B$. Therefore for such an algebra $B$, all elements of $\mathcal{G}$ have non-zero quantum dimension. The representation theory of fusion algebras is well-understood in many cases, hence in all those cases $\mathcal{G}$ can be explicitly determined.

The algebra $B$ of Example 6.2 is a fusion algebra of rational gaussian models of conformal field theory [22]. i $\diamond$

\section{Acknowledgements}

I am grateful to Piotr Hajac and Shahn Majid for discussions and comments. This work was supported by the EU HCM grant and the grant KBN 2 P 30221706 p 01.

\footnotetext{
${ }^{\ddagger}$ I would like to thank Matthias Gaberdiel for a discussion.
} 


\section{References}

1. B. Booss and D.D. Bleecker, Topology and Analysis. The Atiyah-Singer Formula and Gauge-Theoretic Physics. Springer-Verlag, 1985.

2. T. Brzeziński, Differential Geometry of Quantum Groups and Quantum Fibre Bundles. University of Cambridge, $\mathrm{PhD}$ thesis, 1994.

3. T. Brzeziński and S. Majid. Quantum Group Gauge Theory on Quantum Spaces. Commun. Math. Phys., 157 (1993) 591; ibid. 167 (1995) 235 (erratum).

4. R.J. Budzyński and W. Kondracki. Quantum principal fiber bundles: topological aspects. Preprint (1994) hep-th/9401019.

5. C.-S. Chu, P.-M. Ho and H. Steinacker. Q-deformed Dirac monopole with arbitrary charge. Preprint (1994) hep-th/9404023.

6. Y. Doi, Commun. Algebra, 17 (1989) 3053.

7. V.G. Drinfeld. Quantum Groups. In Proceedings of the International Congress of Mathematicians, Berkeley, Cal. Vol.1, Academic Press, 1986, p.798.

8. M. Durdević. Quantum principal bundles. Preprint (1993) hep-th/9311029.

9. L.D. Faddeev, N.Yu. Reshetikhin and L.A. Takhtajan. Quantisation of Lie Groups and Lie Algebras. Leningrad Math. J. 1 (1990) 193.

10. M. Göckeler and T. Schücker. Differential geometry, gauge theories and gravity. Cambridge University Press, 1990.

11. P.M. Hajac. Strong Connections and $U_{q}(2)$-Yang-Mills Theory on Quantum Principal Bundles. Preprint (1994) hep-th/9406129.

12. D. Husemoller. Fibre Bundles. Springer-Verlag, 3rd ed. 1994.

13. D. Kastler. Cyclic Cohomology within Differential Envelope, Hermann, 1988.

14. E. Kunz. Kähler Differentials, Vieweg \& Sohn, 1986.

15. S. Majid. Cross Product Quantisation, Nonabelian Cohomology and Twisting of Hopf Algebras. In. Generalised Symmetries in Physics, H.-D. Doebner, V.K. Dobrev and A.G. Ushveridze, eds., World Scientific, 1994, p. 13.

16. U. Meyer. Projective Quantum Spaces. Lett. Math. Phys. to appear (1994).

17. T. Muir and W.H. Metzler. A Treatise on the Theory of Determinants, Longmans, Green and Co., 1933.

18. M. Pflaum. Quantum Groups on Fibre Bundles. Commun. Math. Phys., 166 (1994) 279.

19. P. Podles. Quantum Spheres. Lett. Math. Phys., 14 (1987) 193.

20. K.-H. Rehren. Markov Traces as Characters for Local Algebras. Nucl. Phys. B (Proc. Suppl.), 18B (1990) 259.

21. M.E. Sweedler Hopf Algebras, Benjamin, 1969.

22. E. Verlinde. Fusion Rules and Modular Transformations in 2D Conformal Field Theory. Nucl. Phys., B300 (1988) 360. 